\title{
El Telecentro UNAH CUROC-Gracias: una práctica innovadora para la equidad educativa universitaria
}

Pedro Antonio Quiel

Centro Universitario Regional de Occidente

Martha Leticia Quintanilla

Dirección de Innovación Educativa

Universidad Nacional Autónoma de Honduras

\section{Resumen}

Desde el año 2010, la Universidad Nacional Autónoma de Honduras (UNAH), al amparo de la política de redes educativas, el modelo de educación virtual, y bajo los principios de equidad, pertinencia y calidad, puso en marcha el proyecto de innovación educativa "Los Telecentros Universitarios".

En el año 2011, el Centro Universitario Regional de Occidente (GUROG), se incorpora a este proyecto en el marco de su

\section{Introducción}

Frente a los cambios tecnológicos acontecidos en las últi- compromiso social de atender las demandas educativas de todos los jóvenes de la red educativa de occidente, dando vida al Telecentro de la UNAH en la ciudad de Gracias, Lempira, con un oferta en modalidad en línea de las carreras de licenciatura en Pedagogía y técnico en Microfinanzas.

Actualmente cerca de 100 estudiantes aprovechan esta oportunidad educativa y han iniciado sus estudios universitarios encaminando sus esfuerzos a culminar con su carrera profesional superior.

La experiencia de innovación educativa y tecnológica de la comunidad académica del CUROC y de la comunidad de Gracias, Lempira se plasma en este artículo.

Palabras Clave: Telecentros, educación superior, equidad, educación en línea, redes educativas. mas décadas, toda la sociedad y la Universidad no es la excep- ción ha tenido que ponerse al día para poder ser más competitiva, 
eficiente y eficaz; pero sin perder de base los principios de igualdad, calidad y pertinencia.

En medio del desarrollo socioeconómico y tecnológico del Siglo XXI, independientemente del nivel de desarrollo de un país o de la sociedad en general, surgen aceleradamente nuevas prácticas o modalidades de educación superior, mismas que han permitido reducir la brecha económica, social, política y cultural entre los diferentes estratos de la sociedad. Es así como se ha pasado de modelos educativos presenciales, a procesos formativos donde el estudiante puede acceder a una oferta educativa utilizando todas las potencialidades de las tecnologías educativas innovadoras.

Estas transformaciones educativas posibilita que muchos jóvenes hondureños que nunca soñaron con estudiar una carrera a nivel superior, vean ahora realizado su sueño; tal es el caso de decenas de estudiantes graduados de educación secundaria en colegios de los distintos municipios de los Departamentos de Lempira, Intibucá y Copán, que hoy aprovechan la presencia de la UNAH a través del Telecentro del CUROC, instalado en la ciudad de Gracias, Lempira.

Las barreras educativas en término de tiempo y espacio, han quedado superadas, con las nuevas modalidades educativas que hacen uso de todas las potenciali- dades educativas que brindan las tecnologías del siglo XXI, y las diversas herramientas de comunicación e interacción sincrónica y asincrónica como audio y video conferencias, teléfonos móviles, correo electrónico, entre otras, para acercar la universidad a los estudiantes.

Los procesos de gestión administrativos y académicos de Universidades como la UNAH, también registran mejoras significativas al aprovechar todo este desarrollo tecnológico. Durante el proceso de Reforma en la UNAH, uno de los cambios más significativos, es que a la luz del modelo educativo que tiene como uno de sus principios la innovación, se inició la puesta en marcha, dando lugar a las primeras carreras en línea ofertadas por la UNAH a través de los Telecentros Universitarios y con ello dando vida a la política de la bimodalidad, que contempla una oferta educativa institucional a nivel presencial, semi-presencial y virtual. No cabe duda que estos procesos innovadores aún imponen muchos desafíos a la Institución.

$\mathrm{Y}$ es aquí donde a partir del 2011 entra en escena innovadora el CUROC, ya que en un esfuerzo de sueños y compromisos compartidos, se logra sumar energías con el gobierno local, fuerzas vivas de la ciudad de Gracias, Lempira, la Vicerrectoría Académica, la Dirección de Innovación Educativa y la Dirección del CUROC, para establecer el primer telecentro de la UNAH en la región de occidente: el Telecentro UNAH CUROC-Gracias, Lempira, ubicado en la ciudad de Gracias, en una parte de lo que hace algunos años constituyó la Escuela Normal. Este telecentro inició el 11 de noviembre del 2011 con el desarrollo de un curso propedéutico para los aspirantes a estudiar bajo esta modalidad y su inauguración oficial se registró el 23 de enero del 2012, la finalidad del mismo es aumentar la cobertura de educación superior en una de las regiones del país más postergadas.

\section{Germen y desarrollo del Telecentro de la UNAH en Gracias}

La UNAH, mediante acuerdo CT-UNAH No. 314-A-207, aprueba las "redes educativas regionales de la UNAH para la gestión del conocimiento con equidad, pertinencia y calidad".

Según el documento de las Redes Educativas de la UNAH (2008), esta nueva visión organizativa de la Institución implica entre otros aspectos:

La reorganización y restructuración de las unidades académicas de la UNAH, poniendo el énfasis en la innovación como eje del proceso gerencial, de cambio y desarrollo; esto significa promover y estimular la generación de ideas, la creatividad individual y colectiva, así como la socialización, sistematización e intercambio de experien- 
cias, lo que requiere de una manera diferente de pensar el Centro o los Centros generadores del conocimiento y de la técnica necesaria para el desarrollo humano sostenible: el Centro está donde están las personas y los grupos que están generando conocimiento y técnica en los diferentes campos. (pp. 30).

Amparado en esta normativa, la CT-UNAH visualizó como parte de la Red Educativa de Occidente, la creación del Instituto Tecnológico de Gracias, y a partir de ello, desde la Vicerrectoría Académica y el CUROC, se impulsaron una serie de acciones, entre ellas la elaboración de un diagnóstico de necesidades educativas de la zona de occidente que posibilitara información científica para responder a las necesidades de desarrollo de la región. Esta actividad se llevó a cabo en conjunto con distintas organizaciones de la sociedad civil de la zona de occidente.

Luego de diversos análisis institucionales y siguiendo lo plasmado en la política de redes educativas y con el propósito de que la UNAH tuviera presencia en Gracias, Lempira, a mediano plazo se iniciaron acciones para conformar el grupo gestor de esa localidad y concretar la creación del Telecentro UNAH CUROC-Gracias, como experiencia institucional y regional, que por un lado atendiera la demanda inmediata de los jóvenes sin posibilidades económicas y sociales para salir de sus comunidades a estudiar en centros presenciales de la UNAH, y por otro lado, que generara experiencia en esta nueva organización de red para que en un segundo momento, bajo los análisis respectivos, continuará el proceso de creación del Instituto Tecnológico.

Una vez constituido el grupo gestor y sentadas las bases para la relación académica y de trabajo colaborativo entre el equipo académico del CUROC, la alcaldía municipal y el grupo gestor de Gracias, Lempira, se estableció a finales del año 2010 e inicios del año 2011 una ruta crítica que permitiera, a través de un esfuerzo conjunto, poner en funcionamiento a finales de ese mismo año el Telecentro de la UNAH en esa ciudad.

En tanto, el modelo de telecentros universitarios de la UNAH, parte de una visión de responsabilidades compartidas, entre la universidad, el gobierno local y las comunidades, la alcaldía de Gracias y el grupo gestor, logró el apoyo financiero del Congreso Nacional, mismo que se tradujo en un aporte de tres millones de lempiras.

Siempre de la mano del gobierno local, el CUROG y la Dirección de Innovación Educativa (DIE) con la participación activa de Secretaría Ejecutiva de Administración de Proyectos de Infraestructura (SEAPI) y la
Dirección Ejecutiva de Gestión de Tecnologías (DEGT), iniciaron y concluyeron, entre mayo y octubre de 2011, los trabajos de acondicionamiento físico y tecnológico del espacio asignado por la alcaldía de Gracias para el funcionamiento del Telecentro. La alcaldía asignó un extremo del edificio colonial donde funcionó la Escuela Normal de Gracias para que se instalara el Telecentro de la UNAH. A la par de los trabajos de acondicionamiento físico se inició el proceso de adquisición del equipo de cómputo, educacional y mobiliario.

Un elemento a resaltar es el trabajo de socialización, tanto del modelo de telecentro como de la oferta de carreras a ofrecer y del proceso de ingreso a la UNAH, que incluye los requerimientos de admisión que implica la aprobación de la Prueba de Aptitud Académica (PAA). Esto demandó la movilización de la Dirección de Admisiones, del equipo académico del CUROC y de miembros del grupo gestor de Gracias, Lempira. Esta actividad es vital, ya que si no se cuenta con alumnos/as que aprueben la PAA, el telecentro no tiene razón de ser.

El telecentro inició funciones en el mes de noviembre de 2011 y fue inaugurado oficialmente por la rectora Julieta Castellanos en febrero de 2012. 
Lo académico: la garantía de la calidad

La UNAH a través del CUROC, nodo central de la red educativa de occidente, llegó a Gracias, Lempira, con un proyecto educativo innovador; ello implicó un cambio de paradigma para todos los actores del proceso formativo y para los mismos pobladores, que imaginaban la presencia de la UNAH a través de una gran edificación física; pero la máxima casa de estudios del país, pertinente al siglo actual llegaba con una oferta educativa mediada por las tecnologías educativas modernas: la Licenciatura de Pedagogía y Ciencias de la Educación y el Técnico en Microfinanzas en la modalidad en línea o virtual.

Los telecentros de la UNAH son espacios tecnológicos y pedagógicos donde los estudiantes pueden tener acceso a educación superior de calidad, a través de la mediación y utilización de tecnologías educativas innovadoras. En ese sentido, con el apoyo de los telecentros, la UNAH puede ofrecer carreras en los distintos niveles en modalidad virtual o en línea.

La UNAH define su educación virtual como el proceso formativo de enseñanza-aprendizaje, donde la relación docente-alumno se encuentra separada en el tiempo y/o en el espacio, durante todo o gran parte del proceso educativo; pero direccionada por una propuesta formativa pedagógica e integral que utiliza soportes, materiales y recursos tecnológicos diseñados especialmente para que los/as alumnos/as alcancen los objetivos de aprendizaje.

El modelo pedagógico de la educación virtual tiene su base en los principios que fundamentan el modelo educativo de la UNAH: calidad, pertinencia, equidad, interdisciplinariedad e internacionalización.

$\mathrm{Su}$ perspectiva tecno-pedagógica apuesta a la innovación como elemento sustantivo para el abordaje y desarrollo de los distintos componentes del proceso formativo, entiéndase currículo, docente, estudiante, metodologías, material didáctico, herramientas tecnológicas de mediación, gestión académico-administrativo y organización institucional.

En ese sentido, llegar a Gracias, Lempira con una oferta educativa en modalidad virtual, implicó para el GUROC una actividad fuerte de capacitación a nivel docente; para que éstos pudieran asumir con soltura su rol como docentes o asesores en línea, con las competencias tecnológicas y pedagógicas que les permitiran ser facilitadores del proceso de aprendizaje de sus estudiantes de forma eficiente. A nivel institucional capacitar docentes para el diseño y desarrollo de ambientes virtuales de aprendizaje, es decir colocar en modalidad en línea todas las asignaturas de la carrera de Pedagogía y el técnico en Microfinanzas.

Asimismo, significó trabajar un proceso de capacitación estudiantil a través de un curso propedéutico, a fin de que ellos también desarrollarán las competencias tecnológicas y pedagógicas para el aprendizaje en línea.

No cabe duda que el éxito de proyectos innovadores como el Telecentro de la UNAH en Gra-

TABLA 1.

\begin{tabular}{|c|c|c|c|}
\hline PERIODO & PEDAGOGIA & MICROFINANZAS & TOTAL \\
\hline I-PAC-2012 & 31 & 1 & 32 \\
\hline III-PAC-2012 & 24 & 6 & 30 \\
\hline I-PAC-2013 & 21 & 2 & 23 \\
\hline III-PAC-2013 & 11 & 3 & 14 \\
\hline TOTALES & 87 & 12 & 99 \\
\hline
\end{tabular}




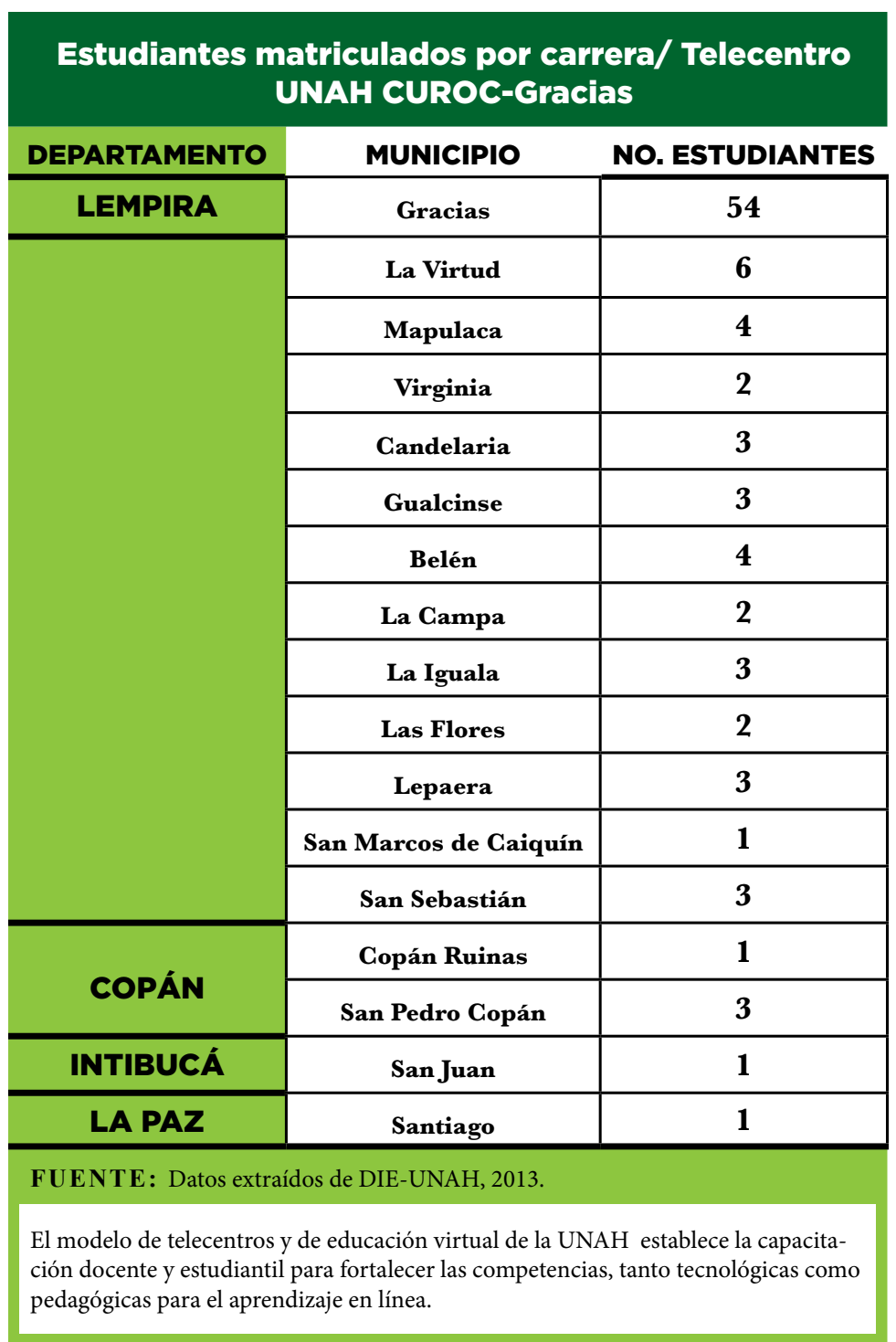

cias, pasa obligadamente por el grado de participación y apropiación de todas las instancias y actores implicados directa o indirectamente, tanto de la UNAH, como de la municipalidad y de las fuerzas vivas de la comunidad, cada uno con sus roles definidos dentro del modelo de telecentros. Será el involucramiento permanente de todos ellos que consolidará las bases para el fortalecimiento y sostenibilidad de esta nueva modalidad de educación superior en la región educativa de occidente.

\section{Resultados}

Para poder valorar el impacto y desarrollo de un telecentro de la UNAH, se deben considerar los principios que rigen los mismos: calidad, pertinencia y equidad. En ese sentido, la UNAH a través de sus redes educativas, está cons-

\section{ciente que no se puede aspirar a matriculas masivas; en tanto la cantidad de estudiantes matricu- lados en un telecentro dependerá, entre otros factores, de la canti- dad de graduados anualmente de secundaria y de que los aspiran- tes que se inscriban para la PAA cumplan con todo el proceso y aprueben la PAA. \\ En ese sentido, consideran- do las características socioeconó- micas del sector de occidente, se resalta que a dos años de funcio- namiento el Telecentro UNAH GUROC-Gracias registre una matrícula de cerca de los 100 es- tudiantes, procedentes de diversos municipios de los departamentos de Lempira, Intibucá y La Paz. En su mayoría los estudiantes es- tán inscritos en la Licenciatura en Pedagogía.}

El impacto en la vida de los actores principales: los estudiantes

Considerando que Honduras muestra cifras dramáticas en su cobertura en educación superior, y pese a que su tasa neta de cobertura reportó un aumento de $3.9 \%$ entre el 2002 y 2010 , esta es la tasa de cobertura más baja en todos los niveles educativos del país, lo que implica que más de 800 mil jóvenes entre 19 y 24 años potenciales estudiantes de las universidades se quedan sin asistir a la educación superior (PNUD, 2012).

De acuerdo a los datos del 
TABLA 3

\begin{tabular}{|c|c|c|}
\hline \multicolumn{3}{|c|}{$\begin{array}{c}\text { Docentes del CUROC capacitados } \\
\text { como asesores en línea }\end{array}$} \\
\hline \multirow{17}{*}{ CUROC } & $\begin{array}{c}\text { UNIDAD } \\
\text { ACADÉMICA }\end{array}$ & CANTIDAD \\
\hline & $\begin{array}{l}\text { Administración } \\
\text { de empresa }\end{array}$ & 1 \\
\hline & Español & 1 \\
\hline & $\begin{array}{l}\text { Historia de } \\
\text { Honduras }\end{array}$ & 1 \\
\hline & Filosofia & 1 \\
\hline & Sociología & 2 \\
\hline & Pedagogía & 3 \\
\hline & $\begin{array}{l}\text { Ciencias } \\
\text { Sociales }\end{array}$ & 1 \\
\hline & Deportes & 1 \\
\hline & $\begin{array}{l}\text { Telecentro } \\
\text { Gracias }\end{array}$ & 2 \\
\hline & $\begin{array}{c}\text { Técnico en } \\
\text { Microfinanzas }\end{array}$ & 5 \\
\hline & Dirección & 1 \\
\hline & Biología & 1 \\
\hline & Letras & 1 \\
\hline & Matemáticas & 3 \\
\hline & $\begin{array}{c}\text { Lenguas } \\
\text { Extranjeras }\end{array}$ & 2 \\
\hline & $\begin{array}{c}\text { Administración } \\
\text { de Empresas }\end{array}$ & 3 \\
\hline
\end{tabular}

PNUD (2012) la tasa neta de cobertura de la educación superior es de $18.7 \%$, lo que se traduce en una matrícula de 110,026 jóvenes de 19 a 24 años en las aulas universitarias que contrasta con los 841,168 hondureños/as potenciales estudiantes, fuera del sistema universitario.

$\mathrm{El}$ análisis de las cifras de acceso a la educación muestra un marcado nivel de exclusión social e inequidad educativa en el nivel educativo superior, de ahí que todo esfuerzo de las universidades del país y especialmente de la UNAH, por llevar la Universidad lo más cerca posible a las comunidades rurales y llegar a los jóvenes que por razones socioeconómicas no están en la UNAH, adquiere un valor superlativo desde la mirada de la equidad educativa.

Desde esta óptica el esfuer- zo de la UNAH para poner en marcha el Telecentro de Gracias, Lempira, se ve reflejada en la esperanza que hoy tienen decenas de jóvenes que valoran la presencia de la UNAH.

En ese sentido, la estudiante de la carrera de Pedagogía Cristina Carabantes expresa:

La educación virtual es un beneficio del cual gozamos en Gracias, Lempira y aprovechamos al máximo esta oportunidad todos los que formamos parte de este proceso educativo. Estamos, como estudiantes, a la vanguardia con la tecnología recibiendo cursos que nos permiten desarrollar todas nuestras actividades de aprendizaje en línea.

En esa misma línea se manifiesta Elmer Raúl Aguirre, también estudiante de la carrera de Pedagogía en el Telecentro UNAH, Gracias, quien se siente orgulloso de hoy ser parte de la UNAH:

Tengo la dicha de poseer esa oportunidad que muchas personas anhelan. Esta experiencia lleva ya dos años y como resultado he adquirido el conocimiento base fundamental para estudiar en esta modalidad. En el curso propedéutico aprendí un estilo de estudio que me ha formado y me ha hecho un estudiante independiente. En secunda- 
ria el curso de computación era una asignatura más, pero estudiar virtualmente está en otro nivel; va ligado al destino de la educación, el cual es ir de la mano con la tecnología, a modo de preparar buenos estudiantes y docentes capacitados para sobrevivir a las demandas que rige la actualidad.

Un testimonio final es el de Clarissa Hernández: "Esta modalidad nos permite como estudiantes organizar nuestro propio tiempo, ser más responsables sin que ningún docente nos esté diciendo lo que tengamos que hacer. Le doy gracias a las personas que han hecho posible la apertura del telecentro, brindando oportunidades a todas las personas del departamento de Lempira".

\section{Conclusiones}

1. Esta nueva práctica innovadora de educación superior abre una ventana de oportunidad a jóvenes de escasos recursos económicos que no tienen la oportunidad de movilizarse a los grandes centros educativos. Parte del éxito de esta nueva práctica se basa en el grado de cohesión y empoderamiento que se dé entre los diferentes sectores de la sociedad, gobierno local, sector privado y la Universidad Nacional.

2. La UNAH a través del Centro Regional Universitario de Occidente, a corto plazo pretende poner en marcha otro telecentro universitario en el departamento de Ocotepeque, el cual vendrá a fortalecer la presencia institucional de la UNAH en la región de occidente, asimismo ofrecer educación superior de calidad.

\section{Referencias}

Universidad Nacional Autónoma de Honduras (2008). Redes Educativas Regionales de la UNAH para la gestión del conocimiento con calidad, pertinencia y equidad (Serie de publicaciones de la reforma universitaria No.2).Tegucigalpa, Honduras.

Universidad Nacional Autónoma de Honduras (2009). Modelo Educativo de la UNAH (Serie de publicaciones de la reforma universitaria No. 3). Tegucigalpa, Honduras.

Universidad Nacional Autónoma de Honduras (2011). El modelo de educación virtual y de Telecentro Universitario de la UNAH. Tegucigalpa, Honduras.

Programa de las Naciones Unidas para el Desarrollo (2012).Informe sobre Desarrollo $\mathrm{Hu}$ mano Honduras 2011. Reducir la inequidad: un desafío impostergable. Honduras. 